\title{
Spontaneous bacterial peritonitis: a prospective Greek multicenter study of its epidemiology, microbiology, and outcomes
}

\author{
Dimitrios N. Samonakis ${ }^{a *}$, Nikolaos Gatselis ${ }^{b *}$, Aristea Bellou ${ }^{b, c}$, Dimitra Sifaki-Pistolla ${ }^{d}$, Maria Melae, \\ George Demetriou'a , Evangelos Thalassinosf, Eirini I. Rigopoulou ${ }^{b}$, Polyxeni Kevrekidouc, \\ Ioannis Tziortziotis ${ }^{\mathrm{d}}$, Kalliopi Azariadib, Melina Kavousanakif, Emmanuel Digenakisa, \\ Themistoklis Vassiliadisc', Elias A. Kouroumalis ${ }^{a}$, George N. Dalekos ${ }^{b}$
}

University Hospital of Heraklion, Crete, Greece; General University Hospital of Larissa, Greece; Papageorgiou General Hospital, Aristotle University of Thessaloniki, Greece; School of Medicine, University of Crete, Heraklion, Crete, Greece; Evangelismos Hospital, Athens, Greece; Venizeleion Hospital, Heraklion, Crete, Greece

Abstract

\begin{abstract}
Background Spontaneous bacterial peritonitis (SBP) is an ominous complication of decompensated cirrhosis. This study aimed to assess several epidemiological, clinical, microbiological and outcome characteristics in Greek patients with SBP, as no solid representative nationwide data of this type was available.

Methods During a 3-year period, 77 consecutive patients with SBP (61 male; median age: 67 years; model for end-stage liver disease [MELD] score: 20), diagnosed and followed in 5 tertiary liver units, were prospectively recruited and studied. Various prognostic factors for disease outcome were studied.

Results Thirty-eight patients had alcohol-related cirrhosis, 17 viral hepatitis, 6 non-alcoholic steatohepatitis, 6 autoimmune liver diseases, and 10 cryptogenic cirrhosis. Hepatocellular carcinoma (HCC) was present in 23 (29.9\%), whereas 10 (13\%) had portal vein thrombosis. The first SBP episode at baseline was community-acquired in 53 (68.8\%), while in 24 (31.1\%) was hospital-acquired, with predominant symptoms abdominal pain and encephalopathy. A positive ascitic culture was documented in $36 \%$ of patients in the initial episode, with almost equal gram (+) and gram (-) pathogens, including 3 multidrug-resistant pathogens. Significant factors for 6-month survival were: higher MELD score, previous $\beta$-blocker use, lower serum albumin, higher lactate on admission and need for vasopressors, while factors for 12-month survival were MELD score and lactate. For overall survival, higher MELD score and lactate along with HCC presence were negative predictive factors.

Conclusions MELD score, lactate, albumin, HCC and treatment with vasopressors were predictive of survival in SBP patients. In hospital-acquired SBP the prevalence of difficult-to-treat pathogens was higher.
\end{abstract}

Keywords Cirrhosis, spontaneous bacterial peritonitis, mortality, lactate, MELD score

Ann Gastroenterol 2021; 34 (1): 1-8
${ }^{a}$ Department of Gastroenterology and Hepatology, University Hospital of Heraklion Crete, Greece (Dimitrios N. Samonakis, George Demetriou, Emmanuel Digenakis, Elias A. Kouroumalis); ${ }^{b}$ Department of Medicine and Research Laboratory of Internal Medicine, National Expertise Center of Greece in Autoimmune Liver Diseases, General University Hospital of Larissa, Greece (Nikolaos Gatselis, Aristea Bellou, Eirini I. Rigopoulou, Kalliopi Azariadi, George N. Dalekos); c c ${ }^{\text {rd }}$ Department of Internal Medicine, Papageorgiou General Hospital, Aristotle University of Thessaloniki, Greece (Aristea Bellou, Polyxeni Kevrekidou, Themistoklis Vassiliadis); ${ }^{\mathrm{d} C l i n i c}$ of Social and Family Medicine, School of Medicine, University of Crete, Heraklion, Crete, Greece (Dimitra Sifaki-Pistolla, Ioannis Tziortziotis); ${ }^{e}$ Department of Gastroenterology, Evangelismos Hospital, Athens, Greece (Maria Mela); 'Department of Internal Medicine, Venizeleion Hospital, Heraklion, Crete, Greece (Evangelos Thalassinos, Melina Kavousanaki) *These authors contributed equally

Conflict of Interest: None

Correspondence to: Dimitrios N. Samonakis, MD, FAASLD, Department of Gastroenterology and Hepatology, University Hospital of Heraklion, Heraklion 71110, Greece, e-mail: dsamonakis@gmail.com

Received 8 November 2020; accepted 20 July 2021;

published online 10 November 2021

DOI: https://doi.org/10.20524/aog.2021.0674

\section{Introduction}

Bacterial infections in patients with cirrhosis are a major clinical problem, as they occur 4-5 times more frequently compared to the general population and can increase mortality by leading to acute-on-chronic liver failure, further decompensation, and multiorgan failure [1-6]. Despite advances in critical care and prophylactic antibiotic use, spontaneous bacterial peritonitis (SBP) is the most common and important bacterial infection in cirrhotic patients with ascites, because of its significant morbidity and mortality $[2,7,8]$.

It is well documented that cirrhotic patients are prone to bacterial infections and SBP, the reasons being multifactorial and associated with a defective immune system, gut dysmotility, alterations in microbiome (intestinal bacterial overgrowth [IBO] and dysbiosis), increased intestinal permeability, and bacterial translocation [9-12]. Previous studies have demonstrated that serum urea, blood leukocyte count, Child-Pugh score and mean arterial pressure are 
independent predictive factors of in-hospital mortality in patients suffering from SBP [13]. Tandon et al showed that model for end-stage liver disease (MELD) score and blood leukocyte count are independent predictors of 30-day mortality [14].

Identifying the patients with SBP likely to present a poor outcome could probably help in the design of strategies to improve prognosis, counsel patients and their relatives on expected outcomes, and establish a follow-up plan. Therefore, we conducted a multicenter prospective study in cirrhotic patients with ascites fulfilling the diagnosis of SBP. Our aim was to study the epidemiological, clinical, laboratory and microbiologic factors that may affect prognosis in Greek patients with SBP, as this type of representative prospective nationwide data was not available.

\section{Patients and methods}

This was a 3-year (October 2014 - October 2017) prospective multicenter study conducted in 5 tertiary liver units from different Greek provinces. During this period, 77 consecutive patients with SBP (61 male; median age: 67.5 years; Table 1) were prospectively recruited and investigated. The study was approved by the local ethics committee of each hospital, and was in accordance with the declaration of Helsinki. Data recorded included patient demographics, clinical information including presenting symptoms, medical comorbidities, presence of hepatocellular carcinoma (HCC), cirrhosis etiology, time from decompensation to SBP development, gastrointestinal bleeding and MELD score, laboratory work-up (ascitic fluid neutrophils, albumin and culture, serum creatinine, albumin and lactate) and drugs used as vasopressors. Detailed data on chronic administration of $\beta$-blockers, proton pump inhibitors (PPIs) and antibiotic prophylaxis for SBP were also recorded. All patients were followed-up until death or transplantation.

The inclusion criteria for the study were: 1) age >18 years; 2) diagnosis of cirrhosis with ascites; 3 ) hospital admission; and 4) informed consent from the patient or from a firstdegree relative, if the patient was encephalopathic. The exclusion criteria were: 1 ) age $<18$ years; 2 ) unwillingness to provide informed consent; 3 ) extrahepatic malignancies; 4) no available data on ascitic fluid; 5) source of infection additional to SBP; and 6) non-cirrhotic portal hypertension.

All patients admitted to the hospital with any of the following underwent abdominal paracentesis on presentation: fever or signs of systemic inflammation, abdominal pain, shock, altered mental status, gastrointestinal bleeding, worsening of liver and/or renal function, or any degree of hepatic encephalopathy. Ascitic fluid was directly inoculated into blood culture bottles (BACT/ALERT ${ }^{\circledast}$ Culture media, BIOMERIEUX, Inc. Durham, NC). The volume inoculated was at least $10 \mathrm{~mL}$ for aerobic and $10 \mathrm{~mL}$ for anaerobic culture, and this was performed at the patient's bedside. Detailed data on cell count, protein and albumin concentration, as well as microbe sensitivities, were collected and analyzed. SBP was diagnosed as an infection of ascitic fluid without any intra-abdominal
Table 1 Demographic, clinical and laboratory characteristics of the participants $(\mathrm{n}=77)$

\begin{tabular}{|c|c|c|c|}
\hline Parameters & Median & IQR & P-value \\
\hline $\operatorname{Sex}^{\star}$ & & & NS \\
\hline Male & 61 & 79.2 & \\
\hline Female & 16 & 20,8 & \\
\hline Age (years) & 67.5 & 14 & NS \\
\hline BMI $\left(\mathrm{kg} / \mathrm{m}^{2}\right)$ & 25.3 & 6.9 & NS \\
\hline $\begin{array}{l}\text { Time from decompensation } \\
\text { (months) }\end{array}$ & 11 & 44 & NS \\
\hline MELD score & 20 & 12 & NS \\
\hline Albumin (g/dL) & 2.8 & 0.69 & NS \\
\hline Lactate $(\mathrm{mmol} / \mathrm{L})$ & 1.8 & 1.8 & 0.06 \\
\hline Urine $\mathrm{Na}(\mathrm{mEq} / \mathrm{L})$ & 22.5 & 31.5 & \\
\hline \multicolumn{4}{|l|}{$\mathrm{BBL}^{*}$} \\
\hline Yes & 52 & 67.5 & \\
\hline No & 25 & 32.5 & \\
\hline \multicolumn{4}{|l|}{$\mathrm{PPI}^{*}$} \\
\hline Yes & 40 & 51.9 & \\
\hline No & 37 & 48.1 & \\
\hline \multicolumn{4}{|l|}{$\mathrm{RXM}^{*}$} \\
\hline Yes & 19 & 24.7 & \\
\hline No & 58 & 75.3 & \\
\hline WBC in ascitic fluid (count) & 1413.5 & 2232 & \\
\hline Albumin duration (days) & 4 & 5 & \\
\hline $\mathrm{VSPR}^{*}$ & & & 0.04 \\
\hline None & 62 & 80.5 & \\
\hline Terlipressin & 7 & 9.1 & \\
\hline Noradrenaline & 7 & 9.1 & \\
\hline Other & 1 & 1.3 & \\
\hline
\end{tabular}

NS, not statistically significant; BMI, body mass index; MELD, model for end-stage liver disease; BBL, $\beta$-blockers; PPI, proton pump inhibitors; RXM, rifaximin; VSPR, vasopressors; WBC, white blood cells

surgically treatable source of infection, and was based on a neutrophil count $>250 / \mathrm{mm}^{3}$ in ascitic fluid, as determined by microscopy or positive culture of the ascitic fluid according to the guidelines of the European Association for the Study of the Liver (EASL) [15]. On admission, all patients had a broad biochemical evaluation, arterial blood gases and baseline liver imaging (ultrasound or computed tomography) to exclude malignancy or other source of infection.

For community acquired SBP, empirical antibiotic therapy with third-generation cephalosporins or piperacilline/ tazobactame and less often with ciprofloxacin, was initiated immediately after diagnosis, without the results of fluid culture. All patients underwent a second paracentesis as a standard approach $48 \mathrm{~h}$ after the initial diagnosis of SBP. Antibiotics were modified to alternative empiric broadspectrum agents if there was no clinical response after $48 \mathrm{~h}$, or changed according to the in vitro susceptibility of isolated organisms once fluid cultures identified the causative agent. For hospital-acquired SBP, patients were mostly commenced on piperacillin/tazobactam or carbapenem with vancomycin. 
Intravenous human albumin was provided to all patients ( $1.5 \mathrm{~g} / \mathrm{kg}$ on diagnosis and $1 \mathrm{~g} / \mathrm{kg}$ on day 3 ) for prevention of the hepatorenal syndrome (HRS); in many patients lower doses of human albumin were continued beyond the third day. HRS was defined according to EASL guidelines [15], after other reasons for renal impairment, such as hypovolemia, shock, parenchymal renal diseases and use of nephrotoxic drugs, had been excluded. HRS was managed with a combination of vasopressor agents (terlipressin or noradrenaline, less frequently octreotide, according to availability) and human albumin infusion, as appropriate.

\section{Statistical analysis}

All variables were tested for normality of distributions by Kolmogorov-Smirnov and binomial test prior to further analysis. Descriptive statistics were estimated for categorical and scale variables, and expressed as median (interquartile range $[\mathrm{IQR}]$ ) and $\mathrm{N}(\%)$, respectively. In addition, chi-square test, Student's $t$-test and Kruskal-Wallis test were employed to assess any potential variations between 6- and 12-month survival and participants characteristics and measurements. Kaplan-Meier survival curves were constructed to assess overall survival, by HCC and etiology, while a multivariate model was developed using Cox regression, testing strata for smoking and adjusting hazard ratios (HR) to age. Two-sided P-values less than 0.05 were considered statistically significant. All data analyses were performed using the statistical software SPSS version 24.0.

\section{Results}

The leading etiology of cirrhosis was alcohol-related, followed by viral, nonalcoholic steatohepatitis (NASH) and autoimmune liver diseases, with a median time of 11 months from decompensation to SBP development (Fig. 1). The median MELD score was $20,77 \%$ of the patients had small (grade I) or large (grade II \& III) varices, while 23 (29.9\%) had HCC of any stage and $10(13 \%)$ had portal vein thrombosis of any grade (Table 1). All cases were first attacks; however, 9 patients developed subsequent episodes. Before the first episode only 9.7\% were under primary prophylaxis for SBP, whereas $68 \%$ of the cohort were receiving $\beta$-blockers and $52 \%$ were on PPIs (Table 1).

In 53 patients the SBP was community acquired. The most frequent clinical presentation was abdominal pain and encephalopathy, but it is notable that in 11 patients (14.3\%) the episode was asymptomatic (Table 2). Nosocomial infections were identified in $24 / 77$ patients (31\%), and similar proportions of the cultures yielded gram $(+)$ or gram (-) pathogens. Symptoms in nosocomial infections were mostly encephalopathy and overt sepsis or liver failure. In $36 \%$ of the first SBP episodes the culture of ascitic fluid was positive (gram (+) 46\%, gram (-) 54\%), with the predominant pathogens being Escherichia coli (E. coli, N=9), Streptococci $(\mathrm{N}=6)$, Staphylococci (aureus and coagulase-negative, $\mathrm{N}=5$ ) followed by Klebsiella spp, Enterococci or other species. Overall, 3 multidrug resistant (MDR) species were identified, namely, E. coli, Enterococcus faecium and Acinetobacter. Other MDR

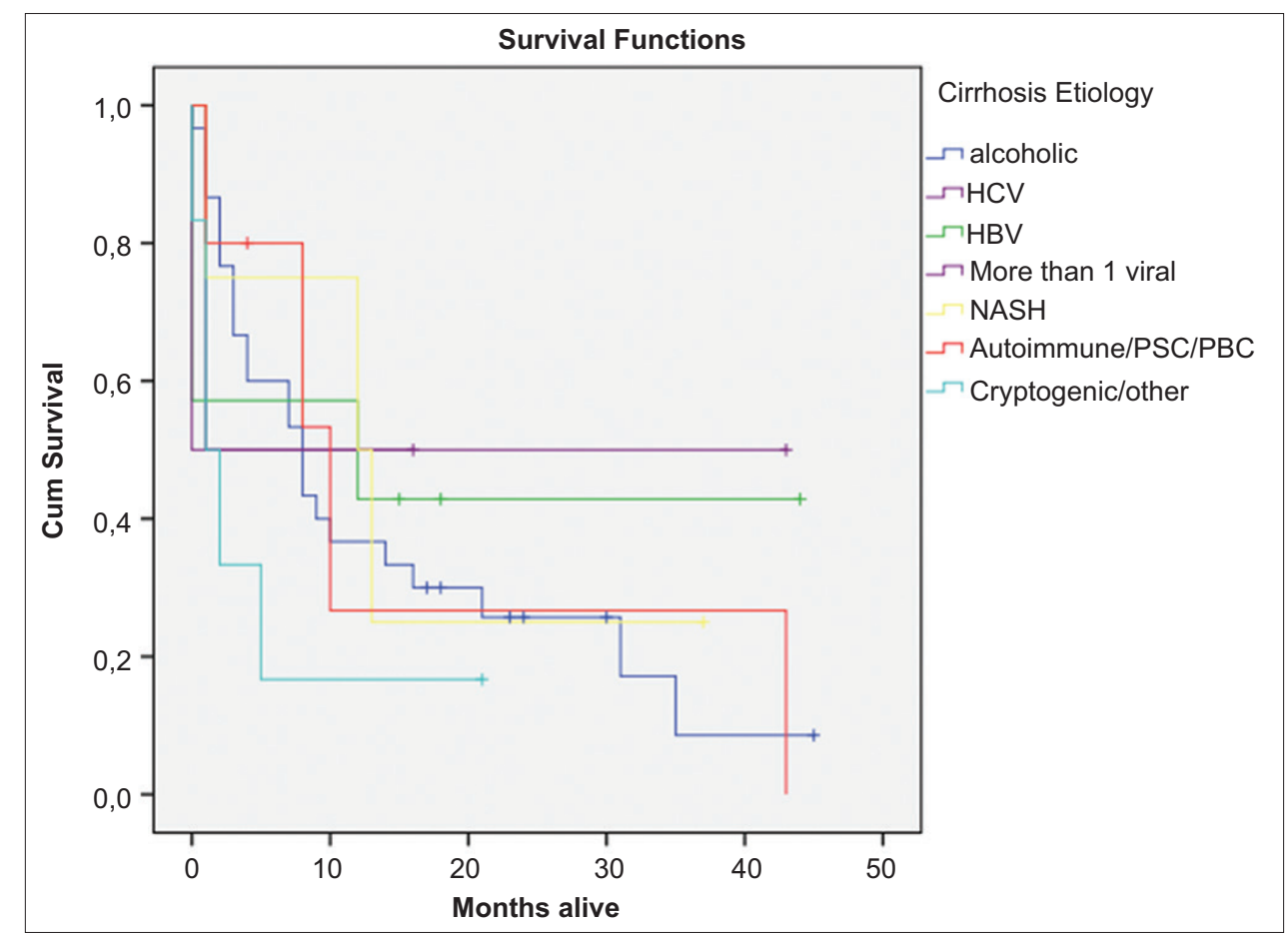

Figure 1 Survival curves according to the etiology of cirrhosis in the SBP study population $H C V$, hepatitis $C$ virus; HBV, hepatitis B virus; NASH, nonalcoholic steatohepatitis; PSC, primary sclerosing cholangitis; PBC, primary biliary cholangitis; SBP, spontaneous bacterial peritonitis 
species (2 E. coli, 2 Enterococci spp, 1 Klebsiella pneumonia, and 1 Staph. aureus) were isolated in subsequent episodes of SBP, all hospital-acquired. Rates of $21 \%$ resistance to third-generation cephalosporins and $14 \%$ resistance to quinolones were identified in those patients with a positive culture in the first episode of SBP. Resistance to third-generation cephalosporins

Table 2 Clinical characteristics of participants

\begin{tabular}{|c|c|c|c|}
\hline Parameters & $\mathrm{N}$ & $\%$ & P-value \\
\hline Diabetes & & & 0.31 \\
\hline No & 50 & 64.9 & \\
\hline Yes & 27 & 35.1 & \\
\hline Alcohol consumption & & & 0.81 \\
\hline Significant & 43 & 55.8 & \\
\hline non-significant & 34 & 44.2 & \\
\hline Smoking & & & 0.39 \\
\hline No & 52 & 67.5 & \\
\hline Yes & 25 & 32.5 & \\
\hline Cirrhosis etiology & & & 0.04 \\
\hline Alcoholic & 38 & 49.3 & \\
\hline $\mathrm{HCV}$ & 4 & 5.2 & \\
\hline HBV & 10 & 12.9 & \\
\hline More than one viral infection & 3 & 3.8 & \\
\hline NASH & 6 & 7.7 & \\
\hline Autoimmune/PSC/PBC & 6 & 7.7 & \\
\hline Cryptogenic/other/unknown & 10 & 12.9 & \\
\hline Hepatocellular carcinoma & & & 0.02 \\
\hline No & 49 & 63.6 & \\
\hline Yes & 23 & 31.2 & \\
\hline Varices & & & 0.06 \\
\hline No & 16 & 20.8 & \\
\hline Small & 35 & 45.5 & \\
\hline Large & 24 & 31.2 & \\
\hline Portal vein thrombosis & & & 0.08 \\
\hline No & 50 & 64.9 & \\
\hline Yes & 10 & 13 & \\
\hline Unknown & 16 & 20.8 & \\
\hline SBP source & & & 0.42 \\
\hline Community & 53 & 68.8 & \\
\hline Hospital acquired & 24 & 31.2 & \\
\hline Gastrointestinal bleeding & & & 0.23 \\
\hline No & 68 & 88.3 & \\
\hline Yes & 24 & 31.2 & \\
\hline Presentation & & & 0.09 \\
\hline Asymptomatic & 11 & 14.3 & \\
\hline Abdominal pain & 32 & 41.6 & \\
\hline Encephalopathy & 18 & 23.3 & \\
\hline HRS & 2 & 2.6 & \\
\hline Shock & 6 & 7.8 & \\
\hline Ascites & 5 & 6.5 & \\
\hline Other & 3 & 3.9 & \\
\hline Patients on primary prophylaxis & & & 0.04 \\
\hline No & 70 & 90.0 & \\
\hline Yes & 7 & 9.1 & \\
\hline
\end{tabular}

HCV, hepatitis C virus; HBV, hepatitis B virus; NASH, nonalcoholic steatohepatitis; $P S C$, primary sclerosing cholangitis; $P B C$, primary biliary cholangitis; SBP, spontaneous bacterial peritonitis; HRS, hepatorenal syndrome and quinolones was similar between gram $(+)$ and gram (-) pathogens. However, resistance to piperacillin/tazobactam was noted only in gram (-) pathogens in our cohort. A change in the antibiotic regimen was necessary in 22 (28.5\%) (3 to piperacillin/tazobactam, 8 to a combination of carbapenems/ vancomycin, and 11 to linezolide, daptomycin or colistin). Twelve of these 22 patients (54.5\%) died within 6 months.

In the study population, the main causes of death were sepsis/multiorgan failure, followed by hepatorenal syndrome, liver failure and HCC progression. In those with nosocomial SBP leading causes were sepsis/multiorgan failure and HRS. The overall survival of the entire cohort was $12.8 \pm 12.9$ months, whereas for the HCC patients it was $7.3 \pm 7.7$ months (Fig. 2). Five patients were lost to follow up. For the alcohol-related cirrhosis, the overall survival was $13.4 \pm 14.6$ months. In the univariate analysis, negative predictors for 6-month survival were: higher MELD score $(\mathrm{P}=0.04)$, previous $\beta$-blocker use $(\mathrm{P}=0.04)$, lower serum albumin $(\mathrm{P}=0.02)$, higher lactate on admission $(\mathrm{P}=0.02)$ and need for vasopressors $(\mathrm{P}=0.01)$ (Table 3). Interestingly, there was no significant association with rifaximin and the use of PPIs. Negative statistically significant predictors for 12-month survival were the MELD score $(\mathrm{P}=0.04)$, the need for vasopressors $(\mathrm{P}=0.01)$ and serum levels of lactate on admission ( $\mathrm{P}=0.03)$. Interestingly, $\mathrm{PPIs}$ had a significant association with 12-month survival (Table 3 ).

In the multivariate analysis, significant factors for overall mortality were: MELD score ( $\mathrm{P}=0.04$; (95\% confidence interval [CI] 1.004-1.091), serum lactate $(\mathrm{P}=0.04$; 95\%CI 1.0551.331), HCC diagnosis $(\mathrm{P}=0.02 ; 95 \% \mathrm{CI} 1.07-3.787)$ and use of vasopressors, i.e., terlipressin ( $\mathrm{P}=0.02$; 95\%CI 1.133-6.969), and noradrenaline ( $\mathrm{P}=0.04 ; 95 \% \mathrm{CI} 1.102-7.361)$ (Table 4).

\section{Discussion}

In this cohort of decompensated cirrhotics, we had the opportunity to study prospectively various characteristics

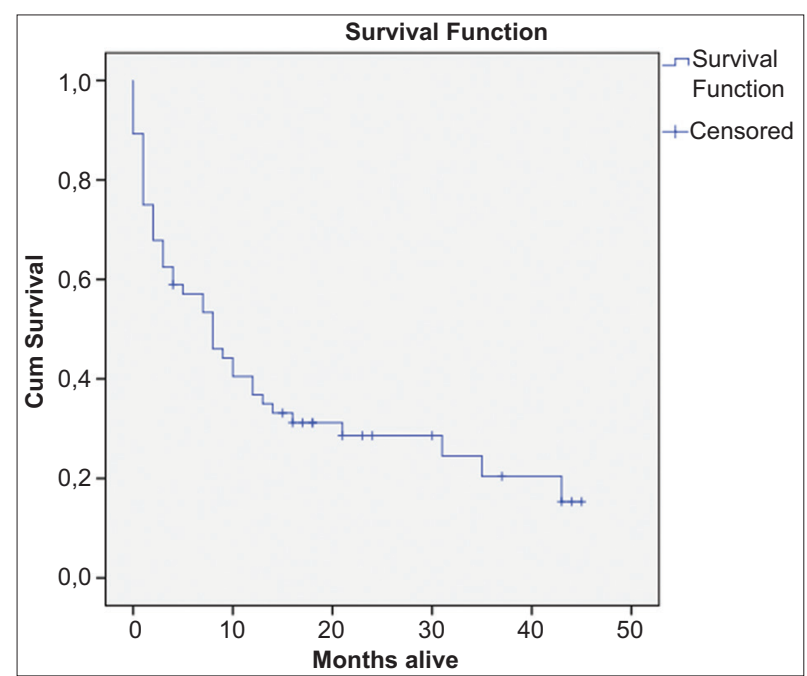

Figure 2 Overall survival curve of the entire population of cirrhotics with spontaneous bacterial peritonitis included in the study 
Table 3 Univariate analysis to identify parameters affecting survival at 6 and 12 months

\begin{tabular}{|c|c|c|c|c|}
\hline \multirow[t]{2}{*}{ Parameters } & \multicolumn{2}{|c|}{ Survival at 6 months } & \multicolumn{2}{|c|}{ Survival at 12 months } \\
\hline & N (\%) & P-value & N (\%) & P-value \\
\hline VSPR & & 0.01 & & 0.01 \\
\hline None & $20(69.9)$ & & $29(76.3)$ & \\
\hline Terlipressin & $6(20.7)$ & & $6(15.8)$ & \\
\hline Noradrenaline & $3(10.4)$ & & $2(5.3)$ & \\
\hline Other & - & & $1(2.6)$ & \\
\hline MELD score ${ }^{*}$ & $17.8(7.1)$ & 0.04 & $18.2(7.2)$ & 0.04 \\
\hline Albumin ${ }^{*}$ & $3(0.5)$ & 0.02 & & \\
\hline Lactate $^{*}$ & $1.9(0.9)$ & 0.02 & $1.9(1.1)$ & 0.03 \\
\hline BBL & & 0.04 & & NS \\
\hline Yes & $52(67.5)$ & & $14(56)$ & \\
\hline No & $25(32.5)$ & & $11(44)$ & \\
\hline PPI & & & & 0.01 \\
\hline Yes & $40(51.9 \%)$ & Ns & $17(48.6)$ & \\
\hline No & 37 (48.1\%) & Ns & $18(51.4)$ & \\
\hline
\end{tabular}

${ }^{*}$ Median (interquartile range)

$V S P R$, vasopressors; MELD, model for end-stage liver disease; $B B L, \beta$-blockers; NS, nonsignificant; PPI, proton pump inhibitors

Table 4 Multivariate analysis ${ }^{\mathrm{a}}$ to estimate risk of mortality

\begin{tabular}{lcccc}
\hline Covariates & $\mathrm{B}$ & $\mathrm{HR}^{*}$ & $95 \% \mathrm{CI}$ & P-value \\
\hline MELD score & 0.04 & 1.04 & $1.004-1.091$ & 0.03 \\
VSPR & & & & \\
None & - & 1 & - & - \\
Terlipressin & 0.80 & 2.81 & $1.133-6.969$ & 0.02 \\
$\quad$ Noradrenaline & 0.32 & 2.23 & $1.102-7.361$ & 0.04 \\
Lactate & 0.17 & 1.18 & $1.055-1.331$ & 0.04 \\
HCC & & & & \\
No & - & 1 & - & - \\
Yes & 0.70 & 2.01 & $1.07-3.787$ & 0.02 \\
\hline
\end{tabular}

aCox regression, ${ }^{*}$ Hazard ratio (HR) has been adjusted to age

$M E L D$, model for end-stage liver disease; VSPR, vasopressors; HCC,

hepatocellular carcinoma; $\mathrm{CI}$, confidence interval

of SBP in Greek patients. Our patient population was fairly representative of Greece, as the participating centers cover cities from the main geographic regions of the country (the island of Crete, Athens, Larissa, and Thessaloniki). We showed that, in Greece: a) SBP patients are quite sick at presentation, as demonstrated by the high median MELD score, and a large number of them have HCC or portal vein thrombosis; b) the first SBP episode is usually community-acquired (almost $70 \%$ ), although a considerable proportion have hospitalacquired infections; c) in the first SBP episode, the isolation of the causative agent was documented in more than a third of patients, with almost equal gram (+) and gram (-) pathogens; d) although relatively few, MDR bacteria are present more frequently in hospital-acquired SBP cases compared to community-acquired ( $25 \%$ vs. $5.7 \%$ ); and e) 6-month survival was associated with no need for vasopressors, lower MELD scores and lower serum lactate levels on admission, higher albumin, and no previous use of $\beta$-blockers, while 12-month survival was associated with lower MELD scores and lactate, and most importantly, the absence of HCC at diagnosis. Overall survival was associated with lower MELD scores and serum lactate, along with no history of HCC and no need for vasopressor use.

The leading etiology of cirrhosis was alcohol-related, followed by viral, NASH and autoimmune liver diseases, in accordance with the etiological stratification of cirrhosis as previously published by our group [16].

As mentioned above, the severity of liver dysfunction, as expressed by a high MELD score, was statistically related to 6-, 12-month, and overall survival from SBP. This is not unexpected, and several publications are in accordance with our data. Schwabl et al [17], in a study of 575 cirrhotics who underwent paracentesis, found a high Child $\mathrm{C}$ score to be a risk factor for developing SBP, and MELD > 22 for high SBPrelated mortality. In a large retrospective study from the Mayo clinic, baseline MELD score predicted the first SBP episode in patients with cirrhosis and ascites [18], while in another report from Germany, intermediate MELD scores were significant for mortality from nosocomial SBP [19]. Moreover, a MELD score $>22$ in patients with SBP was an independent predictor of 30 -day mortality in a retrospective study by Tandon et al [14]. Lower albumin on admission was also significantly associated with worse 6-month survival in our patient cohort. It reflects the severity of liver dysfunction and is frequently affected by the inflammatory process [20].

Another significant factor for survival (6-, 12-month, and overall) was serum lactate levels on admission. Higher lactate was significantly associated with a worse prognosis, as it usually mirrors a more severe infection with more profound tissue hypoxia, more advanced sepsis or imminent septic shock. A study that analyzed patients with septic shock admitted to an intensive care unit (ICU) showed that cirrhotics were more hyperdynamic, had higher plasma lactate concentrations, lower temperature, and a higher mortality rate (compared with patients without cirrhosis) [21]. In a more recent study of 480 consecutive cirrhotic patients admitted to the ICU, lactate levels were associated with higher mortality in those with acute kidney injury [22]. In a retrospective study from Canada, low MELD score and serum lactate on admission were found to be significant for survival in 126 cirrhotic patients with SBPassociated septic shock [23] —findings similar to ours. Renal function was extensively studied, as we tested urea, creatinine, urine sodium and urine volume, at 24 and $48 \mathrm{~h}$; there was no significant association with survival in the univariate analysis.

The need for vasopressors (irrespective of the drug used) was negatively associated with 6-month and overall survival. This finding is reasonable, as these agents were given to cirrhotics admitted with hypotension or evidence of HRS, thus being the most severely ill patients with SBP. Choudhoury et al [24] showed that terlipressin was as effective as noradrenaline in cirrhotics with septic shock, and in addition provided early survival benefit and reduced the risk of variceal bleeding. Lactate clearance was found to be a better predictor of outcome, even after the target of normal mean arterial pressure was achieved, suggesting the role of microcirculation in septic shock [24]. 
The majority of our patients were on $\beta$-blockers for the prevention of portal hypertensive bleeding, whereas only a few were on primary prophylaxis for SBP. This can be explained by the fact that most of the SBP episodes were community acquired. Another confounding factor was that many of our patients had alcohol-related cirrhosis, a population with pure compliance and adherence to any preadmission care. In our study, patients on $\beta$-blockers had a lower 6-month survival rate. This finding could be attributed to their already known advanced portal hypertension. Moreover, patients on $\beta$-blockers occasionally present with hypotension and renal failure in the context of SBP. Our data are in keeping with those published from a retrospective analysis of 607 cirrhotics [25], which showed that, among the patients with cirrhosis and SBP, previous $\beta$-blocker use was associated with a greater proportion of patients being hemodynamically compromised at the time of hospitalization, with higher risk for acute kidney injury or HRS. In contrast, an older meta-analysis had shown that there is a role for $\beta$-blockers in preventing SBP in cirrhotics with ascites, independently of the hemodynamic response [26].

The yield of positive cultures was similar to that reported in the literature and recently by a single Greek tertiary center [27], being most frequent in patients with advanced cirrhosis. There was an equal prevalence of gram (+) and gram (-) bacteria; this is in accordance with many recent studies. In the past, gram (-) bacteria were considered more prevalent in SBP and the choice of antimicrobial treatment was targeted only against these agents. A large retrospective study by Friedrich et al [28] also showed almost equal prevalence of gram (+) and gram (-) bacteria, with third generation cephalosporins to offer a poor microbial coverage. Interestingly, piperacillintazobactam was a very effective treatment in this population. We found relatively few cases with MDR bacteria, and this was almost exclusively in cases with nosocomial SBP. This is in accordance with a prospective study of 312 cirrhotic patients with bloodstream infections, where SBP was associated with a low frequency of MDR organisms [29]. Clearly, the resistance profile of the bacteria causing SBP is different in various parts of the world. Sunjaya et al reported in 481 consecutive patients a quite low yield of positive cultures, but a gram $(+)$ organism was isolated in more than $50 \%$ of the positive cultures. In this cohort there was only $10 \%$ resistance to thirdgeneration cephalosporins [30]. In a retrospective study of 160 SBP cases (35\% positive cultures) in a single transplant center, $45 \%$ of bacterial isolates were resistant to ceftriaxone, notably in patients with hospital-acquired SBP [31]. Recent data from a randomized trial suggests that a combination of more advanced antimicrobials, such as meropenem plus daptomycin, seems more effective than ceftazidime as empiric treatment for nosocomial SBP [32]. The efficacy of empiric treatment was considered as a strong predictor for 90-day mortality. A retrospective series of cirrhotics with SBP reported $42 \%$ gram (+) bacteria, including $17 \%$ MDR bacteria, but importantly resistance to quinolones was 33\% [33]. Furthermore, a prospective study from a single center in our country [34] of 130 cirrhotic patients with SBP or spontaneous bacteremia, showed that the presence of high numbers of drug- resistant isolates was an independent factor for worse outcome. A previous smaller study from the same group showed a very high resistance rate to third-generation cephalosporins (49\%) and quinolones $(47 \%)$, with the majority of isolated bacteria being gram $(+)[35]$.

Fernandez et al reported, in a large prospective series of cirrhotics, that MDR bacteria are mainly isolated in nosocomial infections [36]. However, MDR bacterial infections constitute a prevalent and growing problem in patients with decompensated cirrhosis or acute-on-chronic liver failure, and strategies to prevent the spread of resistance are of paramount importance [37]. A worldwide study of 1302 hospitalized patients with cirrhosis showed a high prevalence of MDR (34\%), with significant geographic variations [38].

The current management of the cirrhotic population including prophylactic treatments with norfloxacin, $\beta$-blockers or other practices, has changed the microbiology of SBP. This is obviously related to the origin of SBP (community or hospital acquired), the local epidemiology and the resistance to widely used antibiotics. The changing epidemiology and bacterial resistance profile demand the knowledge of local epidemiological indices and the implementation ab initio of an empirical effective treatment for SBP.

There has been a lot of debate on the relation of PPIs with SBP in cirrhotic patients. Gastric acid suppression and a rise in gastric $\mathrm{pH}$ facilitate intestinal bacterial overgrowth and increase the risk of bacterial translocation, which can eventually promote the development of SBP. A systematic review and meta-analysis investigating a possible association of gastric acid suppression and the development of SBP found a significant, but quantitatively small association [39]. Dam et al analyzed data from 3 1-year trials of satavaptan for ascites control [40]. The adjusted HR of SBP for current PPI users vs. non-users was 1.72 (95\%CI 1.10-2.69). In contrast, several other studies did not confirm any association with SBP or any other infection or overall mortality [41-44]. PPI use in our cohort had no significant correlation with 6-month survival, whereas for 12 months there was statistical significance. An explanation for this finding is that PPI administration has been associated with gut dysbiosis, which could lead to new events of decompensation, such as hepatic encephalopathy and recurrent episodes of SBP.

Rifaximin is a widely used drug in cirrhotic patients. It is an attractive and safe regimen that has been used in small studies for cirrhotics with SBP. A previous study from our country showed in a small cohort of decompensated cirrhotics that rifaximin was associated with a lower risk of developing complications of portal hypertension, including SBP [45]. By contrast, a larger study failed to show any benefit from the use of rifaximin in relation to the prevention of SBP, despite the fact that the species that caused SBP were altered after rifaximin [46]. The indication for rifaximin in our patients was prevention of hepatic encephalopathy. There was no impact on cultures or resistant pathogens. We did not find any statistical correlation between rifaximin administration and the outcome of SBP.

In conclusion, in this multicenter prospective study of cirrhotics with SBP in Greece, we have shown that MELD score and serum lactate on admission are significant prognostic 
factors for patients' survival. We have also shown that, at least in our hands, vasopressor use, albumin levels and the use of $\beta$-blockers are significant parameters for the outcome of the patients. At the microbiological level, we observed an equal prevalence of gram (+) and (-) bacteria, with a small number of MDR species isolated in hospital-acquired SBP cases. The choice of the appropriate antibiotics should therefore take these factors into account, together with local epidemiology and resistance profile. Larger studies from different countries could provide further important information on this frequent and important complication of cirrhosis.

\section{Summary Box}

\section{What is already known:}

- Spontaneous bacterial peritonitis (SBP) is a significant complication in advanced cirrhosis, with high mortality

- Various factors are implicated in its prognosis

- Recent data have challenged older studies regarding the prevalence of gram (-) bacteria in SBP

\section{What the new finding is:}

- Model for end-stage liver disease score and lactate levels are significant factors for survival in cirrhotic patients with SBP

- Use of vasopressors, $\beta$-blockers and albumin levels were important parameters for SBP outcomes

- There was an equal prevalence of gram $(+)$ and gram (-) bacteria

- Multidrug resistant species were few and were associated with hospital-acquired SBP

\section{Acknowledgment}

We are grateful to Dr Evdoxia Stergiou for her contribution to patient care at Papageorgiou General Hospital

\section{References}

1. Fernandez J, Gustot T. Management of bacterial infections in cirrhosis. J Hepatol 2012;56(Suppl 1):S1-S12.

2. Fernández J, Acevedo J, Castro M, et al. Prevalence and risk factors of infections by multiresistant bacteria in cirrhosis: a prospective study. Hepatology 2012;55:1551-1561.

3. Arvaniti V, D'Amico G, Fede G, et al. Infections in patients with cirrhosis increase mortality four-fold and should be used in determining prognosis. Gastroenterology 2010;139:1246-1256, 1256.e1-e5.

4. Sargenti K, Prytz H, Nilsson E, Kalaitzakis E. Predictors of mortality among patients with compensated and decompensated liver cirrhosis: the role of bacterial infections and infection-related acuteon-chronic liver failure. Scand J Gastroenterol 2015;50:875-883.

5. Moreau R, Jalan R, Gines P, et al; CANONIC Study Investigators of the EASL-CLIF Consortium. Acute-on-chronic liver failure is a distinct syndrome that develops in patients with acute decompensation of cirrhosis. Gastroenterology 2013;144:14261437, 1437.e1-e9.

6. Fernández J, Acevedo J, Wiest R, et al; European Foundation for the Study of Chronic Liver Failure. Bacterial and fungal infections in acute-on-chronic liver failure: prevalence, characteristics and impact on prognosis. Gut 2018;67:1870-1880.

7. Tandon P, Garcia-Tsao G. Bacterial infections, sepsis, and multiorgan failure in cirrhosis. Semin Liver Dis 2008;28:26-42.

8. Wiest R, Krag A, Gerbes A. Spontaneous bacterial peritonitis: recent guidelines and beyond. Gut 2012;61:297-310.

9. Scarpellini E, Valenza V, Gabrielli M, et al. Intestinal permeability in cirrhotic patients with and without spontaneous bacterial peritonitis: is the ring closed? Am J Gastroenterol 2010;105:323-327.

10. Alvarez-Silva C, Schierwagen R, Pohlmann A, et al. Compartmentalization of immune response and microbial translocation in decompensated cirrhosis. Front Immunol 2019;10:69.

11. Arab JP, Martin-Mateos RM, Shah VH. Gut-liver axis, cirrhosis and portal hypertension: the chicken and the egg. Hepatol Int 2018;12:24-33.

12. Jalan R, Fernandez J, Wiest R, et al. Bacterial infections in cirrhosis: a position statement based on the EASL Special Conference 2013. J Hepatol 2014;60:1310-1324.

13. Poca M, Alvarado-Tapias E, Concepción M, et al. Predictive model of mortality in patients with spontaneous bacterial peritonitis. Aliment Pharmacol Ther 2016;44:629-637.

14. Tandon P, Kumar D, Seo YS, et al. The 22/11 risk prediction model: a validated model for predicting 30-day mortality in patients with cirrhosis and spontaneous bacterial peritonitis. Am J Gastroenterol 2013;108:1473-1479.

15. European Association for the Study of the Liver. EASL Clinical Practice Guidelines for the management of patients with decompensated cirrhosis. J Hepatol 2018;69:406-460.

16. Samonakis DN, Koulentaki M, Coucoutsi C, et al. Clinical outcomes of compensated and decompensated cirrhosis: A long term study. World J Hepatol 2014;6:504-512.

17. Schwabl P, Bucsics T, Soucek K, et al. Risk factors for development of spontaneous bacterial peritonitis and subsequent mortality in cirrhotic patients with ascites. Liver Int 2015;35:2121-2128.

18. Khan R, Ravi S, Chirapongsathorn S, et al. Model for end-stage liver disease score predicts development of first episode of spontaneous bacterial peritonitis in patients with cirrhosis. Mayo Clin Proc 2019;94:1799-1806.

19. Kimmann M, Tergast TL, Schultalbers M, et al. Sustained impact of nosocomial-acquired spontaneous bacterial peritonitis in different stages of decompensated liver cirrhosis. PLoS One 2019;14:e0220666.

20. Iliaz R, Ozpolat T, Baran B, et al. Predicting mortality in patients with spontaneous bacterial peritonitis using routine inflammatory and biochemical markers. Eur J Gastroenterol Hepatol 2018;30:786-791.

21. Moreau R, Hadengue A, Soupison T, et al. Septic shock in patients with cirrhosis: hemodynamic and metabolic characteristics and intensive care unit outcome. Crit Care Med 1992;20:746-750.

22. Sun DQ, Zheng CF, Lu FB, et al. Serum lactate level accurately predicts mortality in critically ill patients with cirrhosis with acute kidney injury. Eur J Gastroenterol Hepatol 2018;30:1361-1367.

23. Karvellas CJ, Abraldes JG, Arabi YM, Kumar A; Cooperative Antimicrobial Therapy of Septic Shock (CATSS) Database Research Group. Appropriate and timely antimicrobial therapy in cirrhotic patients with spontaneous bacterial peritonitis-associated 
septic shock: a retrospective cohort study. Aliment Pharmacol Ther 2015;41:747-757.

24. Choudhury A, Kedarisetty CK, Vashishtha C, et al. A randomized trial comparing terlipressin and noradrenaline in patients with cirrhosis and septic shock. Liver Int 2017;37:552-561.

25. Mandorfer M, Bota S, Schwabl P, et al. Nonselective $\beta$ blockers increase risk for hepatorenal syndrome and death in patients with cirrhosis and spontaneous bacterial peritonitis. Gastroenterology 2014;146:1680-1690.e1.

26. Senzolo M, Cholongitas E, Burra P, et al. Beta-blockers protect against spontaneous bacterial peritonitis in cirrhotic patients: a meta-analysis. Liver Int 2009;29:1189-1193.

27. Malli E, Gatselis NK, Dalekos GN, Petinaki E. Combination of vial culture and broad-range PCR for the diagnosis of spontaneous bacterial peritonitis: experience in a Greek tertiary care hospital. New Microbes New Infect 2019;28:1-5.

28. Friedrich K, Nüssle S, Rehlen T, Stremmel W, Mischnik A, Eisenbach C. Microbiology and resistance in first episodes of spontaneous bacterial peritonitis: implications for management and prognosis. J Gastroenterol Hepatol 2016;31:1191-1195.

29. Bartoletti M, Giannella M, Lewis R, et al; ESGBIS/BICHROME Study Group. A prospective multicentre study of the epidemiology and outcomes of bloodstream infection in cirrhotic patients. Clin Microbiol Infect 2018;24:546.e1-546.e8.

30. Sunjaya DB, Lennon RJ, Shah VH, Kamath PS, Simonetto DA. Prevalence and predictors of third-generation cephalosporin resistance in the empirical treatment of spontaneous bacterial peritonitis. Mayo Clin Proc 2019;94:1499-1508.

31. Ardolino E, Wang SS, Patwardhan VR. Evidence of significant ceftriaxone and quinolone resistance in cirrhotics with spontaneous bacterial peritonitis. Dig Dis Sci 2019;64:2359-2367.

32. Piano S, Fasolato S, Salinas F, et al. The empirical antibiotic treatment of nosocomial spontaneous bacterial peritonitis: Results of a randomized, controlled clinical trial. Hepatology 2016;63:1299-1309.

33. Oliveira AM, Branco JC, Barosa R, et al. Clinical and microbiological characteristics associated with mortality in spontaneous bacterial peritonitis: a multicenter cohort study. Eur J Gastroenterol Hepatol 2016;28:1216-1222.

34. Alexopoulou A, Vasilieva L, Agiasotelli D, et al. Extensively drug-resistant bacteria are an independent predictive factor of mortality in 130 patients with spontaneous bacterial peritonitis or spontaneous bacteremia. World J Gastroenterol 2016;22:4049-4056.

35. Alexopoulou A, Papadopoulos N, Eliopoulos DG, et al. Increasing frequency of gram-positive cocci and gram-negative multidrug-resistant bacteria in spontaneous bacterial peritonitis. Liver Int 2013;33:975-981.

36. Fernández J, Acevedo J, Castro M, et al. Prevalence and risk factors of infections by multiresistant bacteria in cirrhosis: a prospective study. Hepatology 2012;55:1551-1561.

37. Fernández J, Prado V, Trebicka J, et al; European Foundation for the Study of Chronic Liver Failure (EF-Clif). Multidrug-resistant bacterial infections in patients with decompensated cirrhosis and with acute-on-chronic liver failure in Europe. $J$ Hepatol 2019;70:398-411.

38. Piano S, Singh V, Caraceni P, et al; International Club of Ascites Global Study Group. Epidemiology and effects of bacterial infections in patients with cirrhosis worldwide. Gastroenterology 2019;156:1368-1380.

39. Khan MA, Kamal S, Khan S, Lee WM, Howden CW. Systematic review and meta-analysis of the possible association between pharmacological gastric acid suppression and spontaneous bacterial peritonitis. Eur J Gastroenterol Hepatol 2015;27:1327-1336.

40. Dam G, Vilstrup H, Watson H, Jepsen P. Proton pump inhibitors as a risk factor for hepatic encephalopathy and spontaneous bacterial peritonitis in patients with cirrhosis with ascites. Hepatology 2016;64:1265-1272.

41. Mandorfer M, Bota S, Schwabl P, et al. Proton pump inhibitor intake neither predisposes to spontaneous bacterial peritonitis or other infections nor increases mortality in patients with cirrhosis and ascites. PLoS One 2014;9:e110503.

42. Hung TH, Tseng CW, Lee HF, Tsai CC, Tsai CC. Effect of proton pump inhibitors on mortality in patients with cirrhosis and spontaneous bacterial peritonitis. Ann Hepatol 2018;17:933-939.

43. Kim JH, Lim KS, Min YW, et al. Proton pump inhibitors do not increase the risk for recurrent spontaneous bacterial peritonitis in patients with cirrhosis. J Gastroenterol Hepatol 2017;32:1064-1070.

44. Terg R, Casciato P, Garbe C, et al; Study Group of Cirrhosis Complications of the Argentine Association for the Study of Liver Disease. Proton pump inhibitor therapy does not increase the incidence of spontaneous bacterial peritonitis in cirrhosis: a multicenter prospective study. J Hepatol 2015;62:1056-1060.

45. Vlachogiannakos J, Viazis N, Vasianopoulou P, Vafiadis I, Karamanolis DG, Ladas SD. Long-term administration of rifaximin improves the prognosis of patients with decompensated alcoholic cirrhosis. J Gastroenterol Hepatol 2013;28:450-455.

46. Lutz P, Parcina M, Bekeredjian-Ding I, et al. Impact of rifaximin on the frequency and characteristics of spontaneous bacterial peritonitis in patients with liver cirrhosis and ascites. PLoS One 2014;9:e93909. 